in some other way? This is what KlapdorKleingrothaus et al. ${ }^{1}$ claim to have done, by observing the nuclear decay ${ }^{76} \mathrm{Ge} \rightarrow{ }^{76} \mathrm{Se} \& 2 \mathrm{e}^{1}$. This reaction is called neutrinoless doubleb-decay, as the final state contains two electrons (historically known as b-particles) and no antineutrinos - so the reaction violates the conservation of lepton number by two units. Taken together with the oscillation measurements, and assuming that the only relevant particles are the three known types of neutrino, the new result implies that the three neutrinos have approximately equal masses, probably a few tenths of an electron volt. This is a surprising result because other particle families, such as quarks and the charged leptons, do not have approximately equal masses (Fig. 1), and it will put a severe constraint on theories of the origin of neutrino masses.

Some caution is called for, however, because of the exceptionally difficult nature of the experiment. Criticisms of the assumptions made by the authors in analysing the background and extracting an extremely small signal have already been offered ${ }^{12,13}$. At any rate, planned future experiments using much larger quantities of ${ }^{76} \mathrm{Ge}$ (or similar nuclei) will achieve much greater sensitivity. By extrapolating from the oscillation measurements, many physicists have guessed, prior to this claim, that a sensitivity $10^{3}$ or $10^{4}$ times greater than that of this experiment may be needed to conclusively observe the violation of lepton-number conservation. Such sensitivity suggests how difficult, as well as how potentially rewarding, future experiments are likely to be.

Edward Witten is at the School of Natural Sciences, Institute for Advanced Study, Olden Lane,

Princeton, New Jersey 08540, USA.

e-mail:witten@ias.edu

1. Klapdor-Kleingrothaus, H. V., Dietz, A., Harney, H. L. \& Krivosheina, I. V. Mod. Phys. Lett. A 16, 2409-2420 (2001). Pati, J. \& Salam, A. Phys, Rev. D 10, 275-289 (1974).

3. Georgi, H. \& Glashow, S. Phys. Rev. Lett. 32, 438-441 (1974).

4. Yanagida, T. in Proc. Workshop on Unified Theory and Baryon Number in the Universe (eds Sawada, O. \& Sugamoto, A.) 95-98 (KEK, Tsukuba, 1979).

5. Gell-Mann, M. et al. in Supergravity (eds van Nieuwenhuysen, $\mathrm{P}$. \& Freedman, D Z) 315-321 (North-Holland, Amsterdam, 1979).

Weinberg, S. in First Workshop on Grand Unification (eds Frampton, P., Glashow, S. L. \& Yildiz, A.) 347-362 (Math. Sci. Press, Brookline, MA, 1980).

Bonn, J. et al. (MAINZ collaboration) Nucl. Phys. Proc. Suppl. 91, 273-279 (2001).

8. Fukuda, S. et al. (SuperKamiokande collaboration) Phys. Rev. Lett. 85, 3999-4003 (2000)

9. Giacomelli, G. \& Giorgini, M. (MACRO collaboration) Preprint hep-ex/0110021 (2001); http://xxx.lanl.gov

10. http://www.sns.ias.edu/ jnb/SNexperiments/experiments.html

11. Bahcall J. N. \& Davis, R. Jr in Essays in Nuclear Astrophysis

(eds Barnes, C. A., Clayton, D. D. \& Schramm, D. N.) 243-285 (Cambridge Univ. Press, 1982).

12. Ferruglio, F., Strumia, A. \& Vissani, F. Preprint hep-ph/0201291 (2002); http://xxx.lanl.gov

13. Aalseth, C. E. et al. Preprint hep-ex/0202018 (2002); http://xxx.lanl.gov

Biomechanics

\title{
Walking with tyrannosaurs
}

Andrew A. Biewener

Tyrannosaurus terrorized the Earth - at least in the Hollywood version of history. But an estimate of the muscle volume in its hind legs suggests that the mighty giant could only walk, not run.

0 ver the course of history, vertebrates have evolved an enormous range of sizes, spanning well over six orders of magnitude in body mass. The largest and most captivating terrestrial giants were the dinosaurs, and Tyrannosaurus - although not the largest at around $6,000 \mathrm{~kg}$ - is perhaps the most famous and terrifying representative of this group. Some workers ${ }^{1,2}$ have argued that bipedal tyrannosaurs and other huge dinosaurs could not move fast because their size would have imposed severe constraints on physiological and mechanical functions. But others claim that these creatures were much more athletic ${ }^{3,4}$.

An obvious difficulty in resolving this argument is that dinosaurs have been extinct for a long time, so reconstructing how they moved is a challenge. But on page 1018 of this issue ${ }^{5}$, Hutchinson and Garcia introduce a new biomechanical approach to the problem, applying an analysis of living animals to their ancient dinosaur relative. They show that Tyrannosaurus simply did not have large

enough leg muscles to produce the forces required for an animal of such size to run.

The skeletal muscles in all animals are made of the same contractile proteins, so their intrinsic capacity for generating force is very nearly the same. The force that can be produced depends on the cross-sectional area of a muscle's fibres. But as body size increases, the geometrical effects of scale mean that muscle capability does not increase proportionately. The force that a muscle can generate increases less rapidly than body weight, so, despite their greater volume, the muscles of larger animals generate less force per unit weight.

In addition, the ability of an animal's skeleton to support mechanical loads decreases with size because bone area does not increase nearly as fast as an animal's weight. Living terrestrial mammals can accommodate these problems of scale by altering their limb posture when they run: larger animals run on more erect limbs than much smaller animals, which gives their muscles greater mechanical advantage ${ }^{6}$ and allows them to maintain similar capacities of force generation and bone loading. But this only applies to animals as large as $300 \mathrm{~kg}$ or so. Above this weight, further changes in muscle mechanical advantage are probably limited ${ }^{7}$, and sustaining force capacity for movement at greater speeds becomes a problem.

So how fast might a $6,000-\mathrm{kg}$ dinosaur have moved? Previous estimates of the speed and locomotive capacity of dinosaurs and other extinct animals have been purely qualitative. Some models are based on the limb motion deduced from the step length and stride frequency derived from fossilized tracks ${ }^{1,2,4,8}$. However, such estimates depend on assumptions about body mass distribution, limb posture and limb length, and about kinematic similarities between species. The data ${ }^{8}$ from fossilized tracks uncovered so far suggest that large bipedal dinosaurs moved at speeds of less than $5 \mathrm{~m} \mathrm{~s}^{11}$. But it may be that tracks left by faster-moving dinosaurs just haven't been discovered yet.

In their analysis of Tyrannosaurus, Hutchinson and Garcia ${ }^{5}$ introduce an approach based on estimates of the minimum muscle mass needed for fast running. First they applied their analysis to alligators and chickens - two living relatives of bipedal dinosaurs. The results show that alligators have less than half the muscle mass that they would need to run fast (if, like bipedal dinosaurs, they used only their hind limbs), whereas chickens have nearly twice the necessary hind-limb muscle mass. This agrees with the observed fact that chickens and many other avian bipeds are good runners, but alligators must support themselves on four limbs and move at relatively modest speeds.

Hutchinson and Garcia then extended their analysis to estimate the limb muscle mass of extinct animals and quantify their locomotive performance. From fossil specimens of Tyrannosaurus, the authors estimated body and segment mass, worked out areas of muscle attachment, and deduced the forces and moments that the creature's leg muscles could have generated. Their analysis rests on assumptions about the limb posture and the magnitude of reaction forces exerted by the ground on the limbs of Tyrannosaurus, and about the kinematic similarity between dinosaurs and living birds and mammals ${ }^{9}$. But their results show that, even if the creature used all its hindlimb muscle mass, it could not have generated the forces necessary for running. They show that for a chicken scaled up to 6,000 kg to run, it would need muscles in each leg equivalent to $99 \%$ of its body mass - which is obviously impossible. The results for smaller bipeds, however, show they probably could run quickly, in agreement with estimates of their speeds from fossil tracks ${ }^{8}$.

A pleasing aspect of Hutchinson and 
Garcia's study is that they apply sensitivity analysis - allowing for a degree of parameter uncertainty - to evaluate the robustness of their results. For instance, they find that the estimated muscle mass is very sensitive to differences in limb posture, but is less sensitive to other parameters, such as muscle-fibre length. Collectively, these uncertainties contribute to up to a threefold variation in the estimated muscle mass for the various models of Tyrannosaurus, but the conclusion is unchanged. Palaeontological analysis of functional performance in fossil organisms will always be an uncertain science, dependent on the availability of fragmentary, long-dead material. So it is welcome when new analytical approaches such as this, and others (such as finite element analysis ${ }^{10}$ ), are brought to bear on such problems.

But what of the reputation that Tyrannosaurus has as a fearsome hunter? Hutchinson and Garcia's results suggest that the creature would have had little success chasing smaller, more fleet-footed prey; it may even have fed on carrion. But I suspect that it could still have moved fast enough to attack other large dinosaurs whose locomotive ability was also limited.

The dinosaurs are famous for being the largest creatures that ever inhabited our planet. But as a group they represent a broad range of size and diversity of form, with a similarly wide range of locomotive capacities and lifestyles ${ }^{11,12}$. It will be interesting to see what insights future investigations of dinosaur diversity yield as new analytical and computational approaches are explored.

Andrew A. Biewener is at the Concord Field Station, Harvard University, Old Causeway Road, Bedford, Massachusetts 01730, USA.

e-mail:abiewener@oeb.harvard.edu

1. Alexander, R. McN. Dynamics of Dinosaurs and Other Extinct

Giants (Columbia Univ. Press, New York, 1989).

2. Farlow, J. O., Smith, M. B. \& Robinson, J. M. J. Vert. Paleontol. 15, 713-725 (1995).

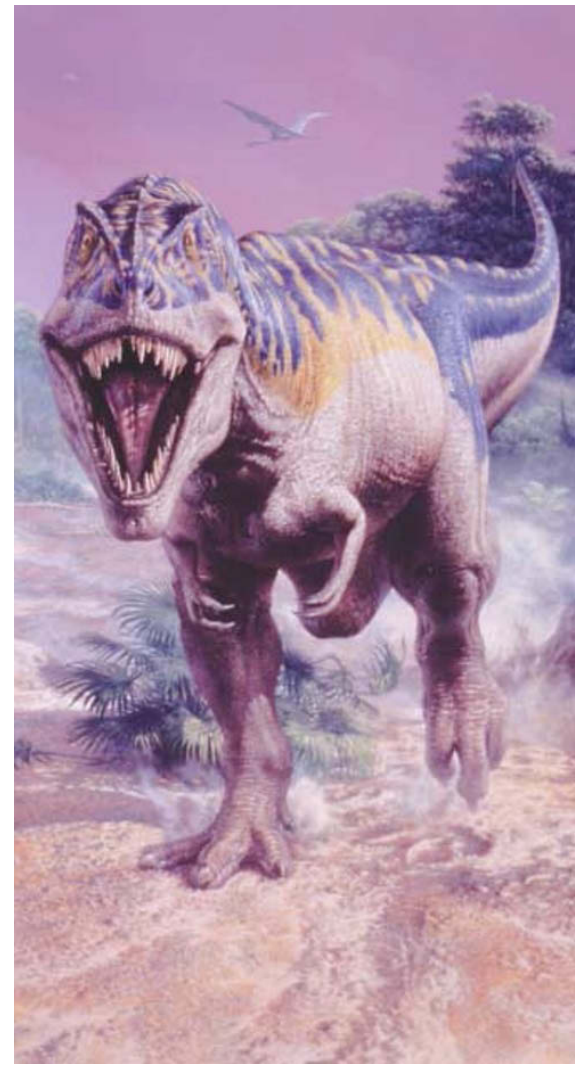

Figure 1 Was Tyrannosaurus as fleet of foot as we thought? Hutchinson and Garcia ${ }^{5}$ analysed the muscle mass and forces in the legs of alligators and chickens, and then extrapolated their results to a $6,000-\mathrm{kg}$ tyrannosaur. Their findings fly in the face of Hollywood legend Tyrannosaurus did not have enough leg muscle to run.

3. Bakker, R. T. Dinosaur Heresies (Willam Morrow, New York, 1986).

4. Paul, G. S. Predatory Dinosaurs of the World (Simon \& Schuster, New York, 1988).

5. Hutchinson, J. R. \& Garcia, M. Nature 415, 1018-1021 (2002)

6. Biewerer, A. A. Science $245,45-48$ (1989).

7. Biewener, A. A. Science 250, 1097-1103 (1990)

8. Farlow, J. O., Gatesy, S. M., Holtz, T. R., Hutchinson, J. R. \& Robinson, J. M. Am. Zool. 40, 640-663 (2000).

9. Alexander, R. McN. \& Jayes, A. S. J. Zool. 201, 135-152 (1983)

10. Rayfield, E. J. et al. Nature 409, 1033-1037 (2001).

11.Carrano, M. T. Paleobiol. 24, 450-469 (1998).

12. Middleton, K. M. \& Gatesy, S. M. Zool. J. Linn. Soc. 128, 149-187 (2000).

\section{Earth science}

\section{Slip-sliding away}

Steven N. Ward

The side of an oceanic volcano, one of the Hawaiian islands, has been caught sliding towards the sea. The distance concerned was only a few centimetres. But it could be an indicator of a huge landslip to come.

S eeing is believing. For Earth scientists especially, the adage holds considerable weight because the 'seeing' is so rare. The geological record tells us that mountain ranges have been built and then washed down to the sea; that entire ocean basins have opened and closed like a door; and that ice a mile thick blanketed the globe a dozen times over. Scientists believe that these events took place, but still, they stuff — the whole side of an oceanic volcano falling into the sea, an event known as flank collapse.

Over time, virtually all oceanic volcanoes grow, become too steep, and slough off flank material. We know this to be true because sonar surveys around most volcanic island chains reveal dozens of old, overlapping debris fields. The Hawaiian islands alone host 70 collapse fields dating from 20 million years ago. Adding up the number of debris fields from all of the ocean's volcanic islands yields the estimate that one flank collapse happens somewhere in the world every 10,000 years on average. Flank collapses are nature's great landslides. Embracing up to $5,000 \mathrm{~km}^{3}$ of rock, they compare to a one-and-a-half-kilometrethick slice of the state of Rhode Island or the island of Majorca racing sideways for 30 or $60 \mathrm{~km}$. In contrast, when Mount Saint Helens erupted in 1980 , only $3 \mathrm{~km}^{3}$ of material blew away.

While flank collapses of oceanic volcanoes are common geologically, none has been caught in action - until now. Cervelli and colleagues' glimpse ${ }^{1}$ of the action came from a network of 20 continuously recording stations of the global positioning system (GPS) scattered about the southeast slope of Kilauea volcano on Hawaii's big island. In November 2000, over a 36-hour period, these GPS stations witnessed a $20-\mathrm{km}$ long and 10-km-wide chunk of the southeast flank move seaward at the speed of 6 centimetres per day. For geophysicists accustomed to tectonic motions of a few millimetres per year, a few centimetres per day is like rocket travel.

To soothe any doubting Thomas, Cervelli et al. spend half of the report reviewing the details of their analysis. The effort is certainly thorough enough to dispel any notion that the signal is a fluke or masquerading noise. For me, their map of a dozen GPS displacement arrows (Fig. 1 on page 1015) all pointing out to sea far beyond their error ellipses tells the whole story. What else can they indicate but some early stage of one of those flank collapses that litter the geological record? A 2,000- $\mathrm{km}^{3}$ piece of Hawaii is slip-sliding away.

In terms of predicting a collapse, the authors interpret their observations more cautiously than I do. By means of dislocation modelling, however, they confirm that the observed GPS displacement field could be explained by $10 \mathrm{~cm}$ of offset on a shallow dipping surface that lies $4.5 \mathrm{~km}$ under their network and that probably extends well out to sea. The offset was a silent earthquake, if you will, on the fault that may eventually detach the whole flank. Thankfully, the November 2000 slide stopped short, but what would it take to dislodge the whole block? Experts believe ${ }^{2-5}$ that intense intrusion of the flank by molten magma dikes 